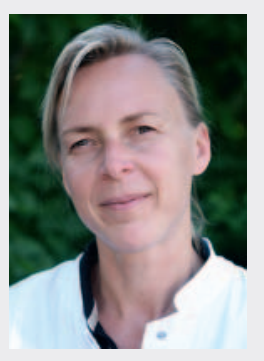

Dr. med. Christina Hauenstein

\section{Grußwort der Kongresspräsidentin}

\author{
Moin, moin in Rostock
}

Moin ist nicht etwa ein muffliger „Guten-Morgen-Gruß“ der manchmal wortkargen Norddeutschen, sondern heißt „Herzlich Willkommen“ und wird dementsprechend auch zu jeder Tageszeit gesagt. So möchte ich Sie herzlich an die Ostseeküste nach Rostock zur 56. Jahrestagung der Gesellschaft für Pädiatrische Radiologie einladen.

Mecklenburg-Vorpommern ist mehr als nur weites Land zwischen Elbe und Ostsee, es lädt zum Verweilen, Erleben, Genießen und Entspannen ein - ob die Rostocker Innenstadt mit ihrem hansestädtischen Charme und der altehrwürdigen Universität oder Warnemünde mit seinen beschaulichen Fischkuttern und Segelschiffen am Alten Strom oder das Meer mit seiner Unendlichkeit, das bei jedem Wetter und zu jeder Jahreszeit seine Schönheit entfaltet.

Wir möchten uns mit unserer Jahrestagung als moderne, klinisch arbeitende Kinderradiologen präsentieren, die eine zentrale Rolle in der interdisziplinären Zusammenarbeit mit allen zugehörigen pädiatrischen Disziplinen spielen.

Am Puls der Zeit werden wir mit Ihnen „Neues“ und „Bewährtes“ auf dem Gebiet der pädiatrischen Neuroradiologie und der muskuloskelettalen Bildgebung inklusive der Kindertraumatologie und Kinder-Rheumatologie diskutieren. Beginnend bei der pränatalen Diagnostik bis hin zu hochspezialisierten interventionellen Verfahren wollen wir einen Einblick in die komplexen radiologischen, diagnostischen und therapeutischen Verfahren geben, um unseren klinischen Partnern geeignete Untersuchungsmodalitäten und auch resultierende therapeutische Maßnahmen zu eröffnen. Mit einer Podiumsdiskussion zum Thema „Kinderschutz, Opferambulanz und gutachterliche Probleme und Fragestellungen“ bei battered child lassen wir am Samstag die Veranstaltung ausklingen.

Im Vorfeld wird es einen ganztägigen Fortbildungskurs für Assistenten in Weiterbildung, pädiatrische Fachärzte und radiologische Kollegen zum Thema Thorax-Bildgebung sowie einen zertifizierten Ultraschallkurs geben. Parallel zum Hauptkongress findet ein ganztägiger praxisnaher Fortbildungstag für MTRA statt.

Mit unserem Motto „Wir setzen Segel in Richtung Zukunft“ freuen wir uns gemeinsam mit Ihnen auf eine spannende, interessante und innovative wissenschaftliche Tagung in Rostock.

Herzlichst,

Dr. med. Christina Hauenstein, Steve Küster und Prof. Dr. med. Sönke Langner

Information und Anmeldung: www.gpr-jahrestagung.de 